\title{
Transition In A Supersonic Boundary Layer Due To Acoustic Disturbances
}

\author{
P.Balakumar \\ Flow Physics and Control Branch \\ NASA Langley Research Center, Hampton, VA 23682
}

\begin{abstract}
The boundary layer receptivity process due to the interaction of threedimensional slow and fast acoustic disturbances with a blunted flat plate is numerically investigated at a free stream Mach number of 3.5 and at a high Reynolds number of $10^{6} /$ inch. The computations are performed with and without two-dimensional isolated roughness element located near the leading edge. Both the steady and unsteady solutions are obtained by solving the full Navier-Stokes equations using the $5^{\text {th }}$-order accurate weighted essentially non-oscillatory (WENO) scheme for space discretization and using third-order total-variation-diminishing (TVD) Runge-Kutta scheme for time integration. The simulations showed that the linear instability waves are generated very close to the leading edge. The wavelength of the disturbances inside the boundary layer first increases gradually and becomes longer than the wavelength for the instability waves within a short distance from the leading edge. The wavelength then decreases gradually and merges with the wavelength for the Tollmien_Schlichting wave. The initial amplitudes of the instability waves near the neutral points, the receptivity coefficients, are about 1.20 and 0.07 times the amplitude of the free-stream disturbances for the slow and the fast waves respectively. It was also revealed that small isolated roughness element does not enhance the receptivity process for the given nose bluntness.
\end{abstract}

\section{Introduction}

Transition from laminar to turbulent state in shear flows occurs due to evolution and interaction of different disturbances inside the shear layer. Though there are several mechanisms and routes to go from a laminar to a turbulent state, most of them generally follow these fundamental processes:

$$
\begin{array}{ll}
\text { - } & \text { Receptivity } \\
\text { - } & \text { Linear instability } \\
\text { - } & \text { Nonlinear instability and saturation } \\
\hline & \text { Secondary instability and breakdown to turbulence }
\end{array}
$$

In the receptivity process, the unsteady disturbances in the environments such as acoustic and turbulence interact with the inhomogeneities in the geometry such as roughness and generate instability waves inside the shear layer. In quiet environments, the initial amplitudes of these instability waves are small compared to any characteristic velocity and length scales in the flow. In the second stage, the amplitudes of these instability waves grow exponentially downstream and this process is governed by the linearized NavierStokes equation. Further downstream, the amplitudes of the disturbances become large and the nonlinear effects inhibit the exponential growth and the amplitude of the waves eventually saturate. In the next stage, this finite amplitude saturated disturbances become unstable to two- and/or three-dimensional disturbances. This is called secondary instability and beyond this stage the spectrum broadens, due to complex interactions and further instabilities, and the flow becomes turbulent in a short distance downstream. In a previous study ${ }^{1}$, the interactions of two-dimensional acoustic disturbances with isolated

1 of 20

American Institute of Aeronautics and Astronautics 
two-dimensional roughness elements in a supersonic boundary layer have been investigated. It was found that isolated roughness does not enhance the generation of disturbances compared to that generated by acoustic disturbances. Since the two-dimensional instability waves are almost neutral, it was difficult to discern between the evolution of the forced continuous spectrum and the instability waves. In this paper, the generation and evolution of three-dimensional disturbances induced by acoustic disturbances and isolated roughness in a supersonic boundary layer with a free stream Mach number of 3.5 is investigated.

Predicting transition onset and transition end points accurately, modeling this transitional region and modeling the turbulence region are the major difficulties in computing the aerodynamic quantities accurately using CFD codes. Our understanding of different instability mechanisms and of different transition processes in shear layers have greatly improved in the last several decades. However transition prediction methods have not made much progress. The main difficulty is due to the nature of the transition process itself. The transition process mainly depends on the boundary layer characteristics and on the frequency and wave number distributions of the disturbances that enter the boundary layer. The laminar boundary layer profiles can be computed easily. The problem is computing, predicting or prescribing the initial spectral, amplitude and phase distribution of the disturbances inside the boundary layer. The initial disturbances are generated by the interaction of the free stream unsteady disturbances and the roughness on the surface. These two are stochastic in nature and are difficult to quantify in general. In any new transition prediction strategy, one should quantify these two quantities and should determine what is the minimum amount of information necessary to predict the transition onset accurately. The objectives of this research work are to answer some of these questions and eventually to come up with an improved transition prediction method.

There have been numerous investigations conducted on the interaction of acoustic waves with supersonic boundary layers. The interactions of acoustic waves with a supersonic boundary layer at finite incident angles using inhomogeneous stability equations are investigated in Ref. 2, 3. One important finding was that due to the interaction, the acoustic waves excite disturbances inside the boundary layer, which are much larger than that in the free stream. The interaction of stream wise acoustic waves with a non-parallel boundary layer was studied in Ref. 4. The analysis and the calculations showed that the disturbances inside the boundary layer reach significant values compared to that in the outside. It was also observed that there exists a critical Reynolds number where this excitation is the highest. Fedorov and Khokhlov ${ }^{5}$ investigated using asymptotic theory the excitation of first and second modes by the acoustic waves near the leading edge region. Gapanov ${ }^{6}$ showed that the energy is transmitted to the T-S waves near the critical region where the ratio between the reflected waves and the incident wave is the largest. There are not many numerical calculations performed to investigate the interaction of acoustic waves with a flat plate including bluntness.

There were several transition experiments performed at NASA Langley in the Mach 3.5 Supersonic Low-Disturbance tunnel. Boundary-layer transition data on a flat plate and on a cone, and free stream noise levels and the power spectral distribution of the free stream noise are presented in Ref. 7. As a first step, the computations are performed for the same conditions as in the experiment. The objectives are to understand the receptivity process near the leading edge of a flat plate and to estimate the receptivity coefficient of the instability waves generated near the leading edge. Computations are also performed to determine whether the slow or the fast acoustic modes are more efficient in generating the instability waves and to estimate the effect of the roughness elements in generating the instability waves when they interact with the acoustic waves. To answer these questions the following computations are performed for a supersonic flow over a flat plate with a blunted leading edge: (1) interaction of a three-dimensional slow acoustic disturbance with the boundary layer, (2) interaction of a three-dimensional fast acoustic disturbance with the boundary layer, and (3) interaction of a three-dimensional slow acoustic disturbance, a two-dimensional roughness element with the boundary layer. A schematic diagram of the computational set up is depicted in Fig. 1.

\section{Governing Equations.}

2 of 20

American Institute of Aeronautics and Astronautics 
The equations solved are the three-dimensional unsteady compressible Navier-Stokes equations in conservation form

$$
\begin{aligned}
& \frac{\partial}{\partial t} Q_{i}+\frac{\partial}{\partial x_{j}}\left(F_{j i}-F_{v j i}\right)=0 .
\end{aligned}
$$

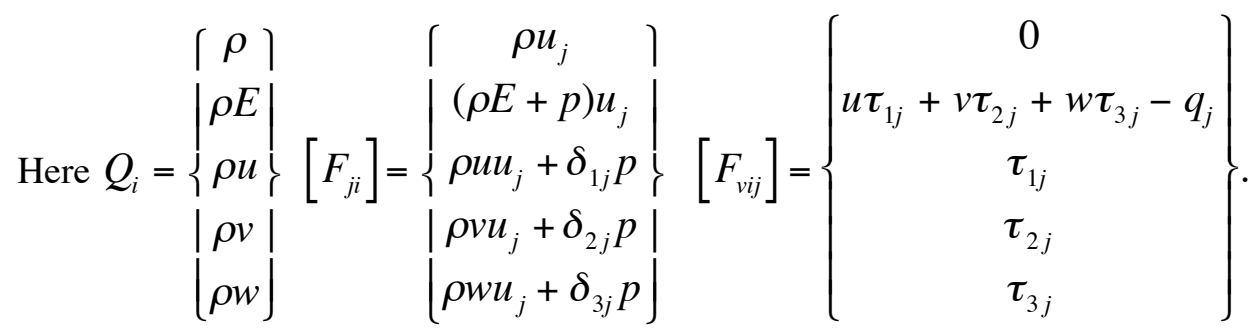

Here $(x, y, z)$ are the Cartesian coordinates, $(u, v, w)$ are the velocity components, $\rho$ is the density, and $p$ is the pressure. $E$ is the total energy given by

$$
\begin{gathered}
E=e+\frac{u^{2}+v^{2}+w^{2}}{2}, \\
e=c_{v} T, p=\rho R T .
\end{gathered}
$$

Here $e$ is the internal energy and $T$ is the temperature. The shear stress and the heat flux are given by

$$
\tau_{i j}=\mu\left\{\frac{\partial u_{i}}{\partial x_{j}}+\frac{\partial u_{j}}{\partial x_{i}}-\frac{2}{3} \delta_{i j} \frac{\partial u_{k}}{\partial x_{k}}\right\}, \quad q_{j}=-k \frac{\partial T}{\partial x_{j}} .
$$

The viscosity $(\mu)$ is computed using Sutherland's law and the coefficient of conductivity $(k)$ is given in terms of the Prandtl number $\operatorname{Pr}$. The variables $\rho, p, T$ and velocity are non-dimensionalised by their corresponding reference variables $\rho_{\infty}, p_{\alpha}, T_{\alpha}$ and $\sqrt{R T_{\infty}}$ respectively. The reference value for length is computed by $\sqrt{v x_{0} / U_{\infty}}$, where $x_{0}$ is a reference location. For the computation, the equations are transformed from physical coordinate system $(x, y, z)$ to the computational curvilinear coordinate system $(\xi, \eta, \zeta)$ in a conservative manner and the governing equations become

$$
\frac{\partial}{\partial t} \bar{Q}_{i}+\frac{\partial}{\partial x_{j}}\left(\bar{F}_{j i}-\bar{F}_{v j i}\right)=0
$$

The components of the flux in the computational domain are related to the flux in the Cartesian domain by

$$
\bar{Q}_{i}=\frac{Q_{i}}{J},\left[\bar{F}_{j i}\right]=\frac{J}{|J|}\left[F_{j i}\right]
$$

where $J=\left\lceil\frac{\partial(\xi, \eta, \xi)}{\partial(x, y, z)}\right]$.

\section{Solution Algorithm}

The governing equations are solved using a 5th order accurate WENO scheme for space discretization and using a third order, total variation diminishing (TVD) Runge-Kutta scheme for time integration. These methods are suitable in flows with discontinuities or high gradient regions. These schemes solve 
the governing equations discretely in a uniform structured computational domain in which flow properties are known point wise at the grid nodes. They approximate the spatial derivatives in a given direction to a higher order at the nodes, using the neighboring nodal values in that direction, and they integrate the resulting equations in time to get the point values as a function of time. Since the spatial derivatives are independent of the coordinate directions, the method can easily add multidimensions. It is well known that approximating a discontinuous function by a higher order (two or more) polynomial generally introduces oscillatory behavior near the discontinuity, and this oscillation increases with the order of the approximation. The essentially nonoscillatory (ENO) and the improvement of these WENO methods are developed to keep the higher order approximations in the smooth regions and to eliminate or suppress the oscillatory behavior near the discontinuities. They are achieved by systematically adopting or selecting the stencils based on the smoothness of the function, which is being approximated. Ref.8 explains the WENO and the TVD methods and the formulas and Ref.9 gives the application of the ENO method to the N-S equations. Ref.10 describes in detail the solution method implemented in this computation.

At the outflow boundary, characteristic boundary conditions are used. At the wall, the simulation uses viscous conditions for the velocities and a constant temperature condition, and it computes density from the continuity equation. In the spanwise direction, symmetric and periodic conditions are used at the boundaries. In the mean flow computations, the simulation prescribes the free-stream values at the upper boundary, which lies outside the bow shock. In the unsteady computations, it superimposes the acoustic perturbations to the uniform mean flow at the upper boundary. The procedure is to first compute the steady mean flow by performing unsteady computations using a variable time step until the maximum residual reaches a small value $\sim 10^{-11}$. These computations use a CFL number of 0.3 . The next step is to introduce unsteady disturbances at the upper boundary of the computational domain and to perform time accurate computations to investigate the interaction and evolution of these disturbances downstream.

The symmetric acoustic field that impinges on the outer boundary is taken to be in the following form.

$$
\begin{aligned}
p_{a c}^{\prime} & =\operatorname{Real}\left\{\tilde{p}_{a c} e^{i \alpha_{a c} x \pm i \beta z+i \varepsilon_{a c} y-i \omega t}\right\} \\
& +\operatorname{Real}\left\{\tilde{p}_{a c} e^{i \alpha_{a c} x \pm i \beta z-i \varepsilon_{a c} y-i \omega t}\right\} .
\end{aligned}
$$

Here $\alpha_{a c}, \beta_{a c}, \varepsilon_{a c}$ are the acoustic wavenumber, and $\omega$ is the frequency of the acoustic disturbance. The wavenumber in the $y$-direction $\varepsilon_{a c}$ determines the incident angle of the acoustic waves and in this paper computations are performed for zero incident angle, $\varepsilon_{a c}=0.0$.

To investigate the effect of roughness element on the transition process, an isolated roughness element of Gaussian shape in the form

$$
f=h e^{-\sigma\left(x-x_{h}\right)^{2}}
$$

is placed at $x=x_{h}$. Here $h$ is the height of the roughness and $\sigma$ determines the width of the roughness in the streamwise direction.

\section{Results}

Computations are performed for a supersonic flow over a semi-infinite flat plate with a blunt leading edge. Table 1 gives the flow parameters and Fig. 1 shows the schematic diagram of the computational set up. The leading edge of the plate is modeled as a super ellipse of the form

$$
\frac{(x-a)^{4}}{a^{4}}+\frac{y^{2}}{b^{2}}=1 \text {. }
$$


Here $b$ is the thickness of the plate and in accordance with the experiment it is taken as 0.0001 inches. It should be noted that the flat plate in the experiment has a beveled leading edge of angle 15 dgrees and the leading edge radius is 0.0001 inches (Fig. 9 in Ref. 7). The aspect ratio $a / b$ is taken as 10 hence the blunt leading edge is joined with the straight portion of the plate at $x=0.001$ in., which is at a Reynolds number of 32.0 .

\section{Table 1 Flow parameters for the wind tunnel model}

Free stream Mach number: $M_{\alpha}=3.5$

Free stream Reynolds number: $R e_{\alpha}=12 * 10^{6} / \mathrm{ft}$

Free stream density: $\rho_{\alpha}=2.249 * 10^{-2} \mathrm{lbm} / \mathrm{ft}^{3}$

Free stream pressure: $p_{\alpha}=187.74 \mathrm{lbf} / \mathrm{ft}^{2}$

Free stream velocity: $U_{\alpha}=2145.89 \mathrm{ft} / \mathrm{s}$

Free stream temperature: $T_{\alpha}=156.42{ }^{\circ} \mathrm{R}$

Free stream kinematic viscosity: $v_{\infty}=1.7882 * 10^{-4} \mathrm{ft}^{2} / \mathrm{s}$

Wall temperature: = adiabatic condition

Prandtl number: $\operatorname{Pr}=0.72$

Ratio of specific heats: $\gamma=1.4$

Length scale $\sqrt{\frac{v_{\infty} x_{0}}{U_{\infty}}}=5.892 * 10^{-5} f t$. $\left(x_{0}=0.5\right.$ in. $)$

The boundary layer thickness at $\mathrm{x}=1$ in.: $\delta_{0}=.01275$ in.

Non-dimensional frequency $F=1 * 10^{-5}$ is equivalent to $41.0 \mathrm{kHz}$

The non-dimensional frequency $F$ is defined as $F=\frac{2 \pi v_{\infty} f}{U_{\infty}^{2}}$,

where $f$ is the frequency in Hertz.

The grid is generated using analytical formulae. The grid stretches in the $\eta$ direction close to the wall and is uniform outside the boundary layer. In the $\xi$ direction, the grid is symmetric about the leading edge and very fine near the nose and is uniform in the flat region. The grid is uniform in the spanwise direction. The outer boundary that lays outside of the shock follows a parabola with its vertex located a short distance upstream of the leading edge of the plate. The computational domain extends from $x=-0.015$ to 12.0 inches in the axial direction. Calculations were performed using a grid size $(3001 * 251 * 11)$. Due to the very fine grid requirement near the nose, the allowable time step is very small and the computations become very expensive to simulate the unsteady computations in the entire domain at once. To overcome this, calculations are performed in two steps. First, the computations are done near the nose region with a very small time step. Second, the flow properties in the middle of the this domain are fed as inflow conditions for the second larger domain and the computations are carried out with a larger time step. The nose region domain extends up to $x=0.65$ in. and the second domain starts at $x=0.3$ inches. Grid refinement studies are performed with different grid distributions near the nose region. Grid 1 is a basic grid of $(801 * 251)$ points with fine grid near the nose and uniform grid in the flat plate region. Grid 2 is obtained by making the domain in the $x$ and y directions half the size. This grid is finer in the shock

5 of 20

American Institute of Aeronautics and Astronautics 
region and in the axial direction. Grid 3 is created to make the grid across the shock finer than Grid 2. Grid 4 is the grid used in the computations with the roughness. This grid has very fine uniform grid near $\mathrm{x}=0.1$ inches where the roughness is located.

\section{Linear instability}

As a prelude for future reference, in Fig. 2 the linear stability results for the similarity boundary layer over a flat plate is presented. The figure depicts the neutral stability diagram in $(R e, F)$ plane for different wave angles 0, 45, 60 and 70 degrees. The figure also shows the N-Factor curves and the growth rates for the most amplified disturbances. The critical Reynolds number is about 193 and this occurs for an oblique wave of angle 60 degrees. The most amplified frequency is about $F=1.0-1.25^{*} 10^{-5}$ and the spanwise wavenumber of the most amplified wave is about $\beta=0.025$. This corresponds to about 0.178 inches in dimensional units and is equivalent to about 14 boundary layer thicknesses. It is also observed that at higher Reynolds numbers $R e>1000$, only the low frequency disturbances $F<3.0^{*} 10^{-5}$ are unstable. This implies that acoustic disturbance with frequencies less than $120 \mathrm{kHz}$ may be the relevant frequency range for generating instability waves inside the boundary layer. The frequency of the most amplified wave is about $40-50 \mathrm{kHz}$ and the maximum $\mathrm{N}$-factor at $x=12$ in. $(R e=3464)$ is about 8.6.

\section{Acoustic waves.}

The linearized Euler equations in a uniform mean flow are:

$$
\begin{aligned}
& \frac{\partial \rho}{\partial t}+U_{0} \frac{\partial \rho}{\partial x}+\rho_{0} \frac{\partial u}{\partial x}+\rho_{0} \frac{\partial v}{\partial y}+\rho_{0} \frac{\partial w}{\partial z}=0, \\
& \rho_{0} \frac{\partial u}{\partial t}+\rho_{0} U_{0} \frac{\partial u}{\partial x}=-\frac{\partial p}{\partial x} \\
& \rho_{0} \frac{\partial v}{\partial t}+\rho_{0} U_{0} \frac{\partial v}{\partial x}=-\frac{\partial p}{\partial y} \\
& \rho_{0} \frac{\partial w}{\partial t}+\rho_{0} U_{0} \frac{\partial w}{\partial x}=-\frac{\partial p}{\partial z} \\
& \rho_{0} c_{p} \frac{\partial T}{\partial t}+\rho_{0} U_{0} c_{p} \frac{\partial T}{\partial x}=\frac{\partial p}{\partial t}+U_{0} \frac{\partial p}{\partial x} \\
& P_{0}=\rho_{0} R T_{0}, \\
& p=\rho_{0} R T+\rho R T_{0} .
\end{aligned}
$$

The solution of this system can be written as 


$$
\left\{\begin{array}{l}
\rho \\
u \\
v \\
w \\
T
\end{array}\right\}=\left\{\begin{array}{c}
\frac{1}{a_{0}^{2}} \\
-\frac{\alpha_{a c}}{\rho_{0}\left(\alpha_{a c} U_{0}-\omega\right)} \\
-\frac{\varepsilon_{a c}}{\rho_{0}\left(\alpha_{a c} U_{0}-\omega\right)} \\
-\frac{\beta_{a c}}{\rho_{0}\left(\alpha_{a c} U_{0}-\omega\right)} \\
\frac{(\gamma-1) T_{0}}{\rho_{0} a_{0}^{2}}
\end{array}\right\} p .
$$

Here the pressure $p$ is in the form

$$
p=\tilde{p}_{a c} e^{i\left(\alpha_{a c} x+\varepsilon_{a c} y+\beta_{a c} z-\omega t\right)}
$$

The dispersion relation among the wavenumbers $\alpha_{a c}, \beta_{a c}, \varepsilon_{a c}$ and the frequency $\omega$ is given by

$$
\left(\alpha_{a c} U_{0}-\omega\right)^{2}=\left(\alpha_{a c}{ }^{2}+\beta_{a c}{ }^{2}+\varepsilon_{a c}{ }^{2}\right) a_{0}^{2} .
$$

For zero incident angle $\varepsilon_{a c}=0$, the wavenumber $\alpha_{a c}$ can be expressed as

$$
\alpha_{a c}=\frac{\omega \cos \theta_{z}}{\left(U_{0} \cos \theta_{z} \pm a_{0}\right)}
$$

Here $\theta_{z}=\tan ^{-1}\left(\frac{\beta_{a c}}{\alpha_{a c}}\right)$ is the sweep angle and the plus sign corresponds to the fast moving wave and the minus sign corresponds to the slow moving wave. The corresponding phase speeds are $C=U_{0} \pm \frac{a_{0}}{\cos \theta_{z}}$. The wavenumber for the fast moving wave is $\alpha_{a c}<\frac{\omega}{U_{0}+a_{0}}$ and for the slow moving wave $\alpha_{a c}>\frac{\omega}{U_{0}-a_{0}}$ and the sweep angle is limited by $\theta_{z}<\cos ^{-1}\left(\frac{1}{M}\right)$. At $M=3.5, F=1.25^{*} 10^{-5}$ the wavenumbers of the fast moving and slow moving waves are $\alpha_{a c}<0.00687$ and $\alpha_{a c}>0.01237$ and the sweep angle of the slow moving wave is limited to 73.39 degrees. Table 2 gives the wavenumbers and the wavelengths in dimensional units for the slow, the fast acoustic waves and for the neutral stability waves for $\beta_{a c}=0.0$ and 0.025 . 
Table 2 Values of $\alpha_{\underline{a c}}$ and wavelength for different sweep angle

\begin{tabular}{|c|c|c|c|}
\hline$\beta_{a c}$ & Slow & Fast & $\begin{array}{c}\text { Neutral } \\
\text { wave }\end{array}$ \\
\hline 0.0 & 0.01237 & 0.00687 & 0.01225 \\
& $(0.359$ in. $)$ & $(0.646$ in. $)$ & $(0.362$ in. $)$ \\
\hline 0.025 & 0.01757 & 0.00168 & 0.01502 \\
& $(0.253$ in. $)$ & $(2.644$ in. $)$ & $(0.296$ in. $)$ \\
\hline
\end{tabular}

The variation of the wavenumber $\alpha$ with the Reynolds number $\sqrt{\frac{U_{\infty} x}{v_{\infty}}}$ for a two-dimensional $\beta=0.0$ and a three-dimensional $\beta=0.025$ instability waves are plotted in Fig. 3. The wavenumber for the twodimensional wave decreases from 0.0124 at a Reynolds number of 220 to 0.0119 at a Reynolds number of 2000. For the three-dimensional disturbance it decreases from 0.01775 at a Reynolds number of 190 to 0.01285 at a Reynolds number of 2000. The Reynolds numbers at the neutral points are 820 and 610 respectively for the two and three-dimensional disturbances and they are also marked in Fig. 3. The figure also depicts the range of the acoustic wavenumber $\alpha_{a c}$ for the two and three-dimensional slow acoustic waves. It is seen that perfect matching in the wavenumbers occur at very low Reynolds number range where the disturbances are marginally stable. For the unit Reynolds number of $1.0 * 10^{6} / \mathrm{in}$., Reynolds number of 200 is located at .04 inches from the leading edge of the plate and the neutral point is located close to $x=0.36$ inches. Hence there may be strong generation of instability waves in the nose region where the bluntness effects and the non-parallel effects will become important.

\section{Mean flow.}

Figure 4 shows the mean flow density contours computed using the WENO code. Figure 4a shows the entire domain and Fig. 4b shows the flow field near the nose region. The outer boundary of the leading edge shock is located approximately at 0.00003 inches from the nose, which is $1 / 6^{\text {th }}$ of the leading edge thicknesses upstream. Figures 4(c) and (d) similarly show the density contours of the mean flow with the roughness element. The Mach wave originated from the roughness element propagates parallel to the Mach wave originated from the leading edge. The density profiles for the case without roughness at $x$ $=0.01,0.05,0.25,0.5,1.0,3.0$ inches $\left(\sqrt{\operatorname{Re}_{x}}=100,224,500,707,1000,1732\right)$ are plotted in Fig. 5a. Figure $5 \mathrm{~b}$ shows the same profiles in the similarity coordinates. The Blasius similarity profile is also included for comparison. It is seen that very close to the leading edge, there exists a strong shock and this compression expands over the leading edge and the shock becomes weaker away from the nose region. The boundary layer profiles slowly approach the similarity profiles close to $x=0.5$ inches.

Figure 6(a) shows the comparison between the growth rate and $\mathrm{N}$-factors computed using the similarity profiles and the profiles obtained from the numerical simulation for the most amplified wave with $F=1.25 * 10^{-5}$ and $\beta=0.025$. Figure $6 \mathrm{~b}$ shows the second derivative of the temperature for the two mean profiles at the station $x=5.0$ inches. It is seen that the stability results obtained using the simulation profiles agree reasonably good with that is obtained using the similarity profiles.

\section{Interaction of three-dimensional acoustic waves with the boundary layer.}

After the mean flow is obtained, three-dimensional slow and fast acoustic disturbances are separately introduced at the outer boundaries and time accurate simulations are performed. The non-dimensional frequency and the spanwise wavenumber are $F=1.25^{*} 10^{-5}$ and $\beta=0.025$. These parameters give the largest $\mathrm{N}$-factor close to the experimental transition location. To remain in the linear regime, very small 
initial amplitude of $\tilde{p}_{a c} / p_{\infty}=1.0 * 10^{-7}$ is prescribed for the free-stream acoustic waves. Even with these small initial amplitudes, nonlinearity starts to develop near the end of the computational domain $x \sim 10.0$ inches.

Figure 7 shows the results for the evolution of the unsteady fluctuations obtained from the simulation for the slow wave at a fixed time while Fig. 8 shows the disturbances for the fast wave. Figures 7(a), 8(a) show the contours of the density fluctuations in the entire domain and Figs. 7(b), 8(b) depict the results inside the boundary layer. The perturbation field can be divided into four regions. One region is the area outside the shock where the acoustic waves propagate uniformly. The second region is the shock layer across which the acoustic waves are transmitted. The third region is the area between the shock and the boundary layer. This region consists of transmitted external acoustic field and the disturbances that are radiated from the boundary layer. The fourth region is the boundary layer where the boundary layer disturbances evolve. The figures show that the disturbances inside the boundary layer originate at the nose region. As these disturbances evolve downstream, the wavelength decreases as is clearly seen in the first 0.50 inches in Fig. 7(b). As noted earlier, the wavelength of the fast acoustic wave is about ten times longer than that for the slow wave. An interesting observation is the appearance of short wavelength disturbances inside the boundary layer starting from the nose region. Figure 9 shows the $u$-velocity fluctuation in the $y-z$ plane and the results are plotted for one wavelength in the spanwise direction. The disturbance is concentrated near the critical layer and as expected follows a cosine waveform in the spanwise direction.

As described earlier, grid refinement studies have been performed with different grid distribution near the nose region. Figure 10 shows the pressure distribution along the wall obtained with different grids. Figure 11 shows the eigenfunction distributions near the wall and across the shock very near the nose region $x=0.01$ and 0.20 inches. It is seen that all three computations give exactly the same results, except the shock becomes thinner with the fine grids, and confirm that the results obtained are not due to any numerical resolutions.

Figure 12 shows the instantaneous pressure fluctuations along the wall for the slow and the fast waves and Fig. 13 shows the amplitude of the pressure fluctuations along the wall in a log scale. Figure 13 also includes the results from the parabolized stability equations (PSE) computations obtained for the same mean boundary layer profiles. The figures clearly show the initial generation and the eventual exponential growth of the instability waves inside the boundary layer. The slow wave whose wavelength is closer to the wavelength of the instability wave transforms into instability waves smoothly. The fast waves whose wavelengths are much larger are initially modulated by short wavelength disturbances. These short waves transform into instability waves and grow exponentially downstream. The growth of the disturbances agrees very well with the PSE results about one acoustic wavelength downstream of the neutral point. Following the PSE results up to the neutral point, the initial amplitude of the instability waves at the neutral point can be estimated. From these values the receptivity coefficients defined by the initial amplitude of the pressure fluctuations at the wall at the neutral point non-dimensionalised by the freestream acoustic pressure can be evaluated.

$$
C_{\text {recpt }, p_{\text {wall }}}=\frac{\left(p_{\text {wall }}\right)_{n}}{p_{a c}}
$$

The computed receptivity coefficient for the slow and the fast waves are

$$
\begin{aligned}
& C_{\text {recpt }, p_{\text {wall }}, S}=1.2 \\
& C_{\text {recpt }, p_{\text {wall }}, F}=0.07
\end{aligned}
$$

Similarly, the receptivity coefficients based on the maximum density fluctuations inside the boundary layer normalized by $\rho_{a c}$ are calculated.

$$
\begin{gathered}
C_{r e c p t, \rho_{\max }, S}=8.4 \\
C_{\text {recpt }, \rho_{\max }, F}=0.47 \\
9 \text { of } 20
\end{gathered}
$$


The ratio of the receptivity coefficient between the slow and the fast modes are about 17.0. As expected, the slow modes whose phase speed are close to the neutral stability waves of the boundary layer excite the instability waves more efficiently than the fast waves.

Figure 14 shows the wavenumber variation along the streamwise direction calculated from the unsteady fluctuations along the wall. This is achieved by first decomposing the fluctuations in to harmonic components of the form

$$
\begin{aligned}
p(x, z, t) & =\tilde{p}(x) e^{i \beta z-i \omega t}+c . c \\
& +\tilde{p}(x) e^{-i \beta z-i \omega t}+c . c .
\end{aligned}
$$

The wavenumber of the fluctuations are evaluated from the expression

$$
\begin{aligned}
\alpha(x) & =\left(\frac{1}{i \tilde{p}(x)} \frac{\partial \tilde{p}(x)}{\partial x}\right) \\
& =\alpha_{r}+i \alpha_{i}
\end{aligned}
$$

Figure 14a shows the wavenumber distributions for the whole domain and Fig. 14b shows the results near the nose region for the slow wave. The figure includes the wavenumber distributions obtained from PSE variations computations, linear stability computations. The wavenumber of the slow acoustic wave is also drawn in the figure. Beyond the initial nose region $x>0.3$ in., the numerical wavenumber distribution agrees very well with the pse and the linear stability results. The interesting part is how the slow acoustic wave with a higher wavenumber interacts and merges with the instability waves inside the boundary layer. This is clearly illustrated in the Fig. 14b. The wavenumber near the nose region first decreases slowly up to $x \sim 0.1$ in., it then increases slowly for $x>0.1 \mathrm{in}$. and merges with the T-S wave around $x \sim$ 0.3 inches.

Figure 15 shows the eigenfunction distributions obtained from the simulation and the linear stability computations for the density fluctuations at different axial locations. Figure 15(a) shows the distributions near the nose region and Fig. 15(b) shows the results in downstream region. It is seen that away from the nose region the computed eigenfunctions agree with the linear stability results and confirms the previous conclusions that the generated disturbances are in fact the T-S waves. Near the nose region the eigenfunctions differ from that for the instability waves and this region represents the interaction region.

Figure 16 shows the evolution of pressure fluctuations along the wall for the cases with and without the isolated roughness element. The computations are performed for $h=0.0002$ in., $\sigma=5.0$ and $x_{h}=0.1$ in. This gives $h / \delta=1 / 20$ and the width of the roughness equals to about two boundary layer thicknesses at $x=x_{h}$. There are no noticeable differences between the two results implying that the small isolated roughness elements do not enhance the instability waves and the instability waves are basically generated by the interaction of acoustic wave with the leading edge flow field. The variation of the wavenumber computed using Eq. (18) is also included in Fig. 14. It is interesting to see that the wavenumber follows the same variation as in the no roughness case except near the roughness and confirm the conclusion that the roughness does not affect the receptivity process at these small roughness heights.

\section{Discussion and Conclusions}

The boundary layer receptivity process due to the interaction of three-dimensional slow and fast acoustic disturbances with a blunted flat plate and the evolution of disturbances inside the boundary layer are numerically investigated at a free stream Mach number of 3.5 and at a high Reynolds number of $10^{6} /$ inch. The computations are performed with and without two-dimensional isolated roughness element located near the leading edge. Both the steady and unsteady solutions are obtained by solving the full Navier-Stokes equations using the $5^{\text {th }}$-order accurate weighted essentially non-oscillatory (WENO)

10 of 20

American Institute of Aeronautics and Astronautics 
scheme for space discretization and using third-order total-variation-diminishing (TVD) Runge-Kutta scheme for time integration. At this Reynolds number, the neutral stability region occurs within about one to two acoustic wavelengths from the leading edge.

The mean flow computations show that the leading edge shock is located upstream at about $1 / 6^{\text {th }}$ of the thickness of the plate and the strength of the shock weakens within a short distance downstream. It is also noted that the boundary layer profiles approach the similarity profiles within $x \sim 0.5$ to 1.0 inches from the leading edge. The linear stability and the N-Factor computations show that the stability characteristics of the computed and the similarity profiles are almost the same.

The simulation of interaction of three-dimensional plane slow and fast acoustic waves with the boundary layer shows that the instability waves are generated very close to the nose region. The receptivity coefficients computed based on the pressure fluctuations at the wall at the neutral point are about 1.20 and 0.07 for the slow and fast acoustic waves respectively. They are about 8.40 and 0.47 based on the maximum density fluctuations at the neutral point. This implies that the slow waves are more efficient, about 17 times, in generating instability waves inside the boundary layer than the fast waves.

The wavenumber distributions computed from the fluctuations inside the boundary layer revealed that it initially decreases gradually starting from the leading edge and becomes even smaller than the wavenumber for the instability waves. It then increases in downstream and merges with that for the instability waves. The disturbances inside the boundary layer induced by the long wavelength fast acoustic waves are modulated by short wavelength disturbances starting from the leading edge. These short waves initiate the instability waves in downstream.

The simulation results for the amplification of disturbances inside the boundary layer agree very well with the PSE results downstream of the neutral point. This implies that beyond the initial region, the external forcing does not enhance or modify the amplitude of the instability waves. Simulation of the interaction of slow acoustic waves with an isolated two-dimensional roughness shows that small roughness elements do not enhance the amplitude of the instability waves.

The above findings suggest that in two-dimensional supersonic boundary layers, the slow acoustic waves are the main catalyst in causing the transition. By knowing the approximate receptivity coefficients and the spectrum of the free-stream slow acoustic disturbances, the initial amplitudes of the instability waves can be approximately prescribed and linear and nonlinear PSE computations can be performed to predict the transition onset points accurately.

\section{References}

1. P. Balakumar, "Transition in a Supersonic Boundary layer Due to Roughness and Acoustic Disturbances," AIAA Paper 2003-3589, 2003.

2. Mack, L. M., "On the application of linear stability theory and the problem of supersonic boundary-layer transition," AIAA Journal., 13, No. 3, 1975.

3. Gaponov, S. A., "Interaction between a supersonic boundary layer and acoustic disturbances," Fluid Dynamics, 6, 1977.

4. Gaponov, S. A. and Smorodsky, B. V., "Supersonic Boundary Layer Receptivity to Streamwise Acoustic Field," IUTAM Symposium,, Spinger-Verlag, 1999.

5. Fedorov, A. V., and Khokhlov, A. P., " Excitation of Unstable Modes in a Supersonic Boundary Layer by Acoustic Waves,” Fluid Dynamics, No. 9, pp. 456-467, 1991.

6. Gaponov, S. A., "Excitation of Instability Waves in the Supersonic Boundary Layer by Sound," IUTAM Symposium Potsdam, Springer-Verlag, 1993.

7. Chen, F. J., Malik, M. R., Beckwith, I. E., "Comparison of Boundary Layer transition on a Cone and Flat Plate at Mach 3.5," AIAA-88-0411, 1988.

11 of 20

American Institute of Aeronautics and Astronautics 
8. Shu, Chi-Wang, “ Essentially Non-Oscillatory and Weighted Essentially Non-Oscillatory Schemes for Hyperbolic Conservation Laws," NASA/CR-97-206253 and ICASE Report N0. 976

9. Atkins, H. L., "High-Order ENO Methods for the Unsteady Compressible Navier-Stokes Equations," AIAA Paper 91-1557, 1991.

10. Balakumar, P., Zhao, H., and Atkins, H., "Stability of Hypersonic Boundary_layers Over a Compression Corner," AIAA Paper 2002-2848, 2002. 


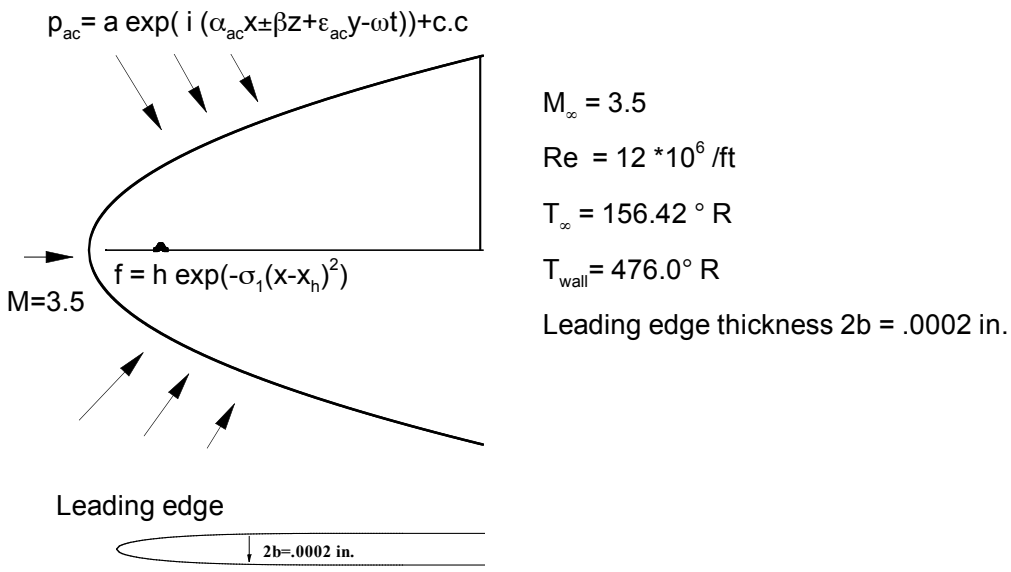

Figure 1. Schematic diagram of the computational model.
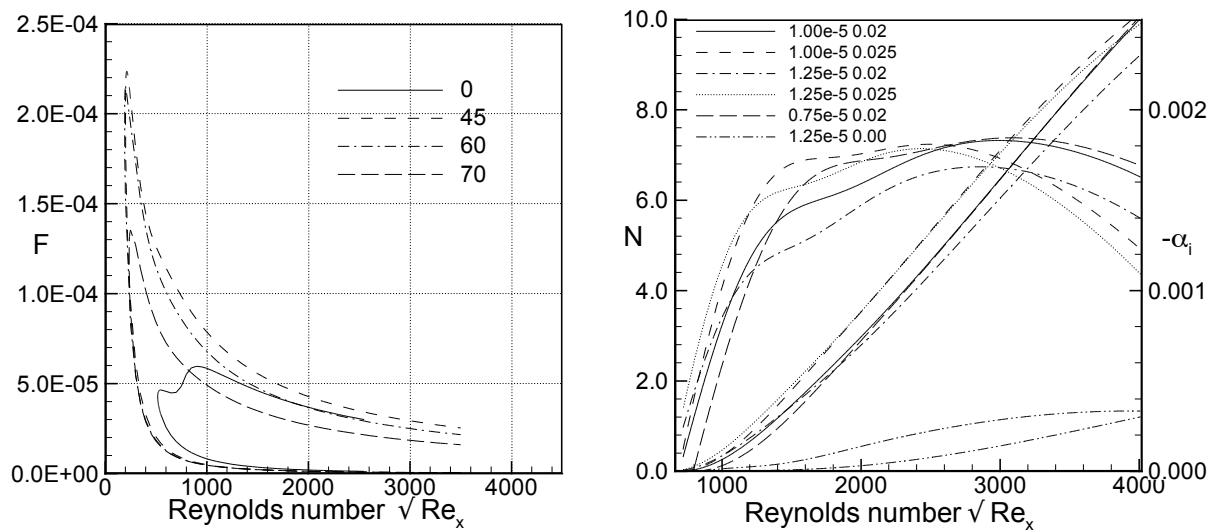

Figure 2. Stability and N-Factor diagrams for a flat-plate boundary layer. $M_{\infty}=3.5, T_{\infty}=80^{\circ} \mathrm{F}$

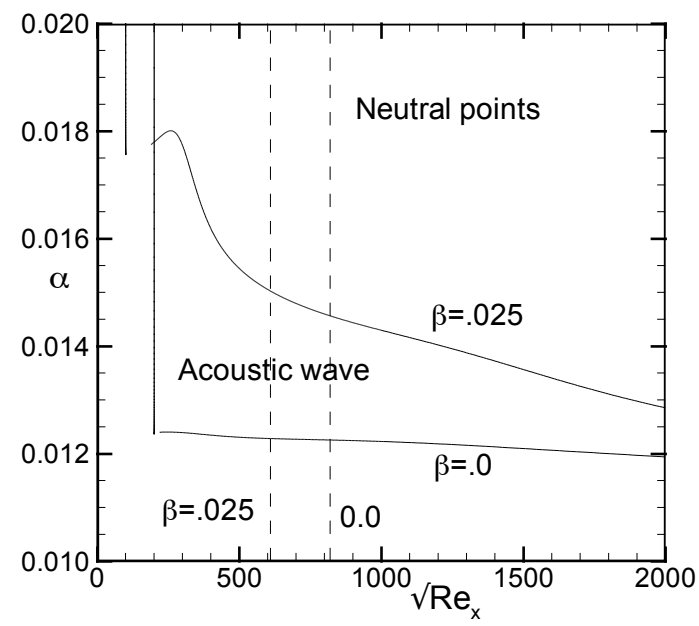

Figure 3. Variation of the wavenumber with the Reynolds number for the instability waves and the wavenumber for the acoustic waves for $\beta=0.0$ and 0.025 .

13 of 20

American Institute of Aeronautics and Astronautics 
(a)

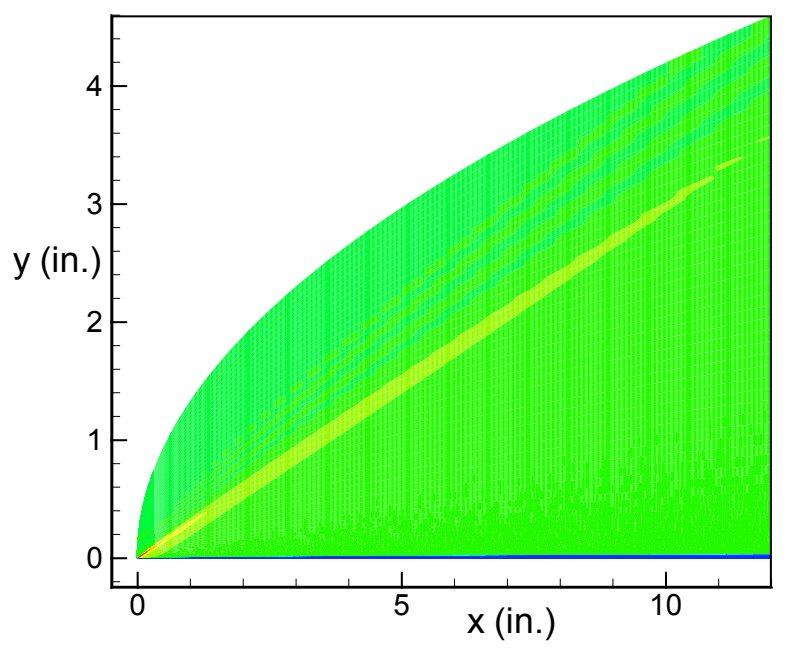

(c)

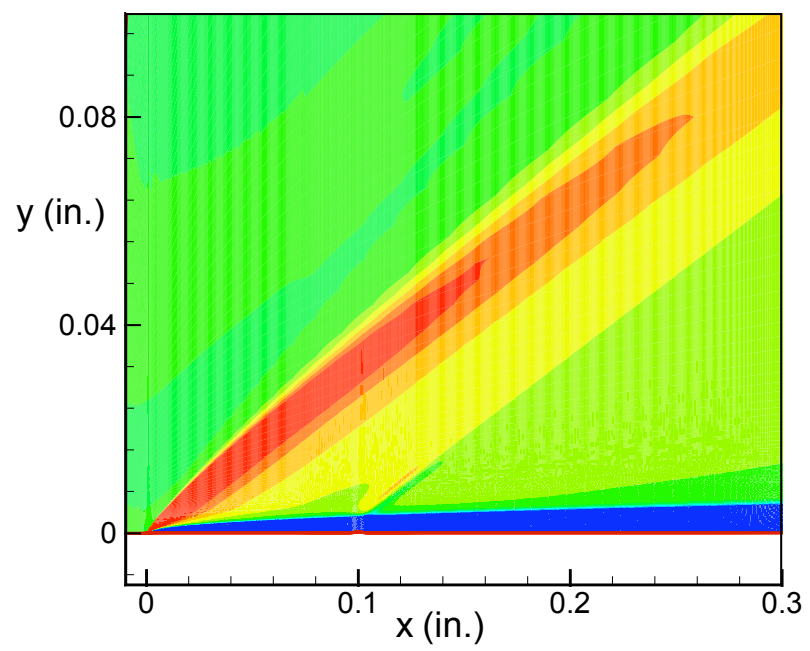

(b)

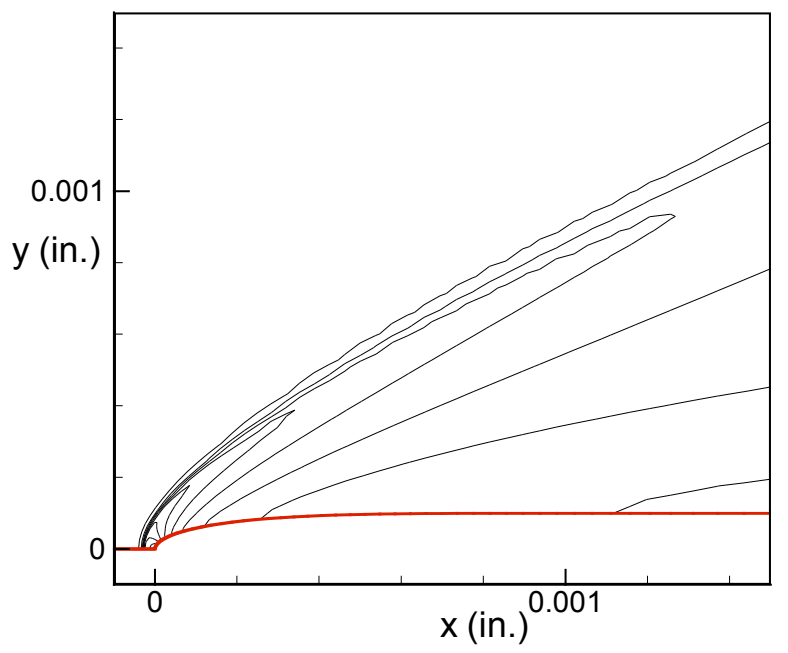

(d)

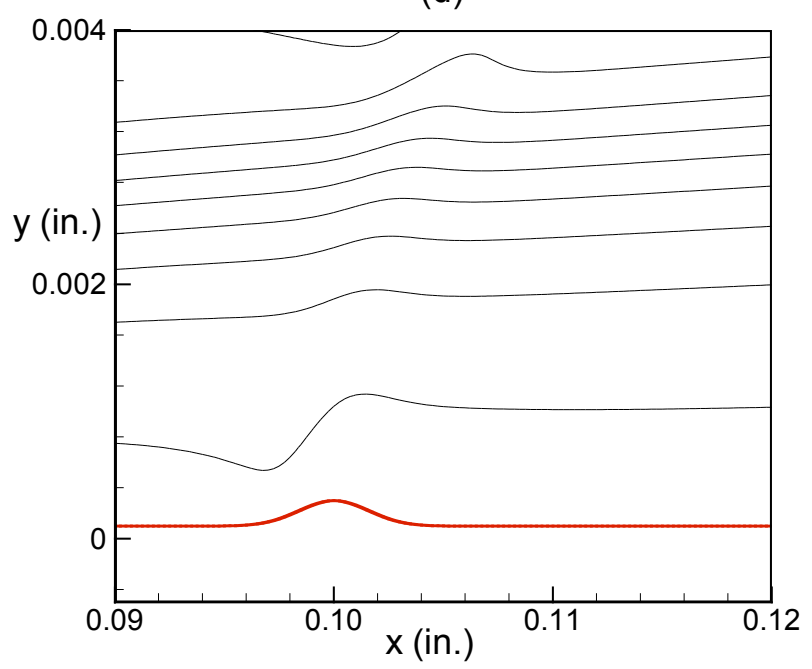

Figure 4. Contours of the density for flow over a flat plate with a blunted leading edge at $M=3.5$ with and without roughness. $h=0.0002$ in., $x_{h}=0.1$ in. 

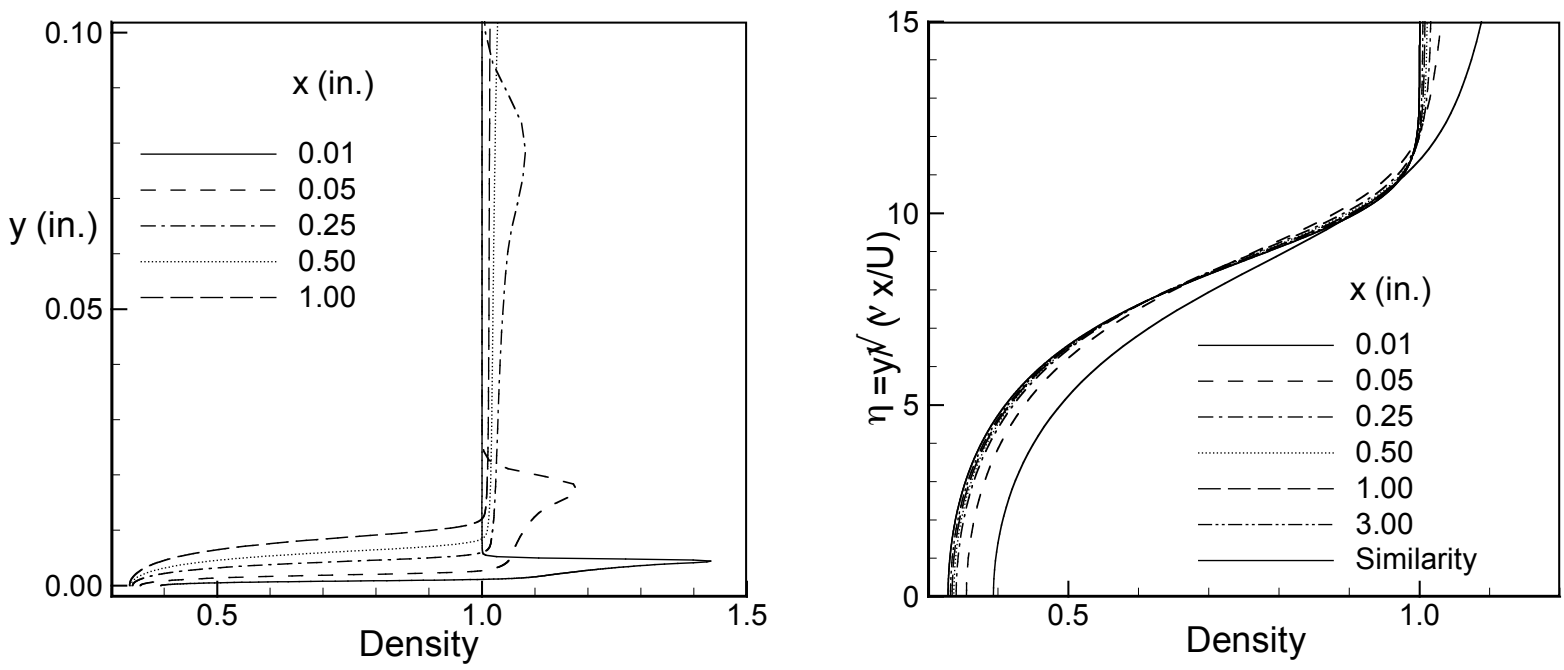

Figure 5. Mean density profiles at different $x$ locations.
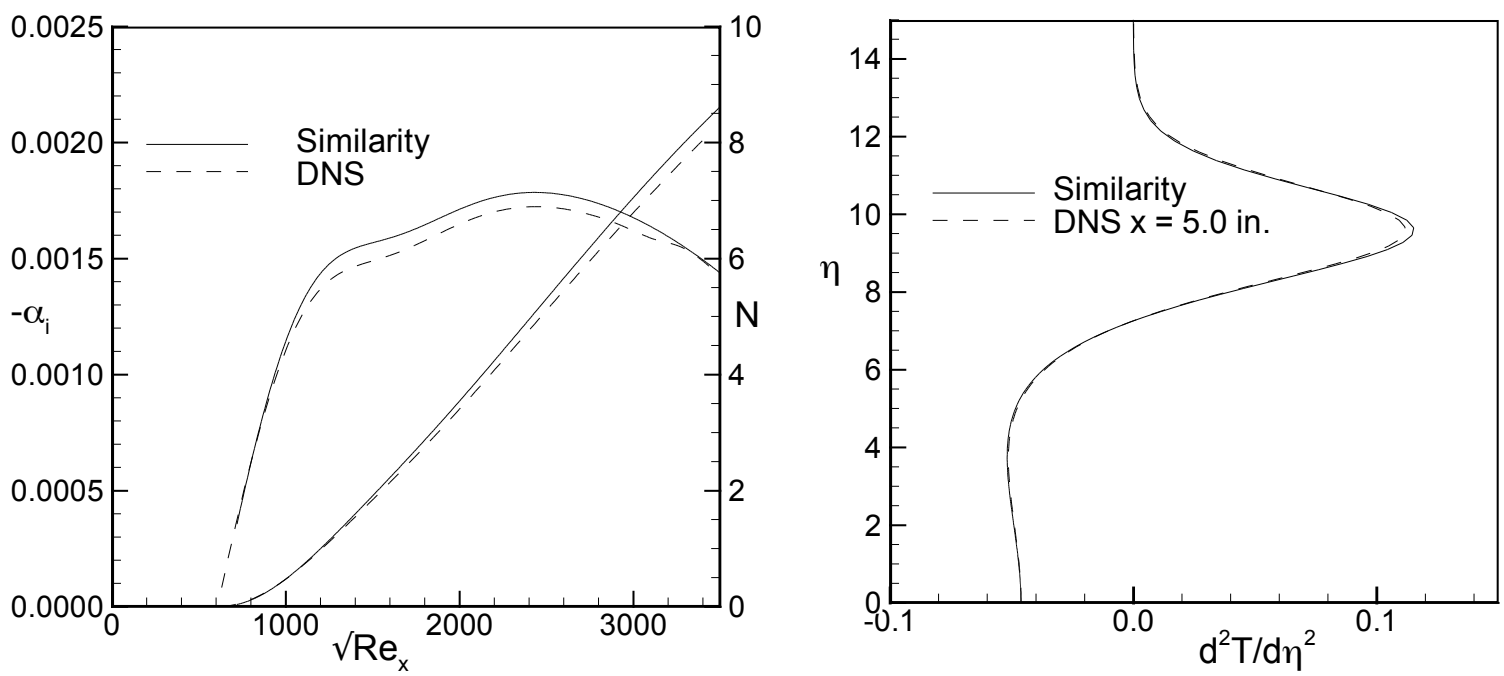

Figure 6. (a) Comparison between the linear stability results computed using the similarity profiles and the profiles from the numerical solution. (b) Comparison of second derivatives for the temperature. 

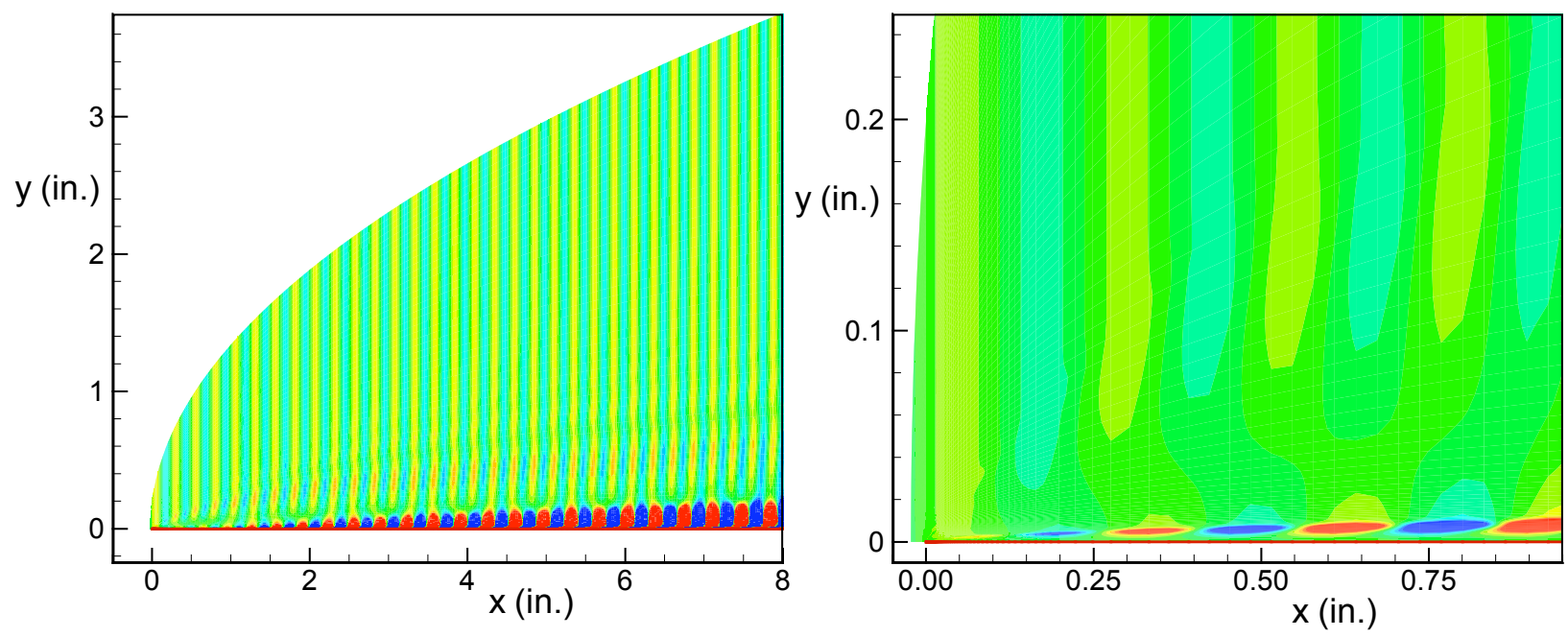

Figure 7. Contours of the unsteady density fluctuations due to the interaction of a 3D slow acoustic wave with a flat plate with a blunted leading edge. $F=1.25 * 10^{-4}, \beta=0.025$, incident angle 0.0 .
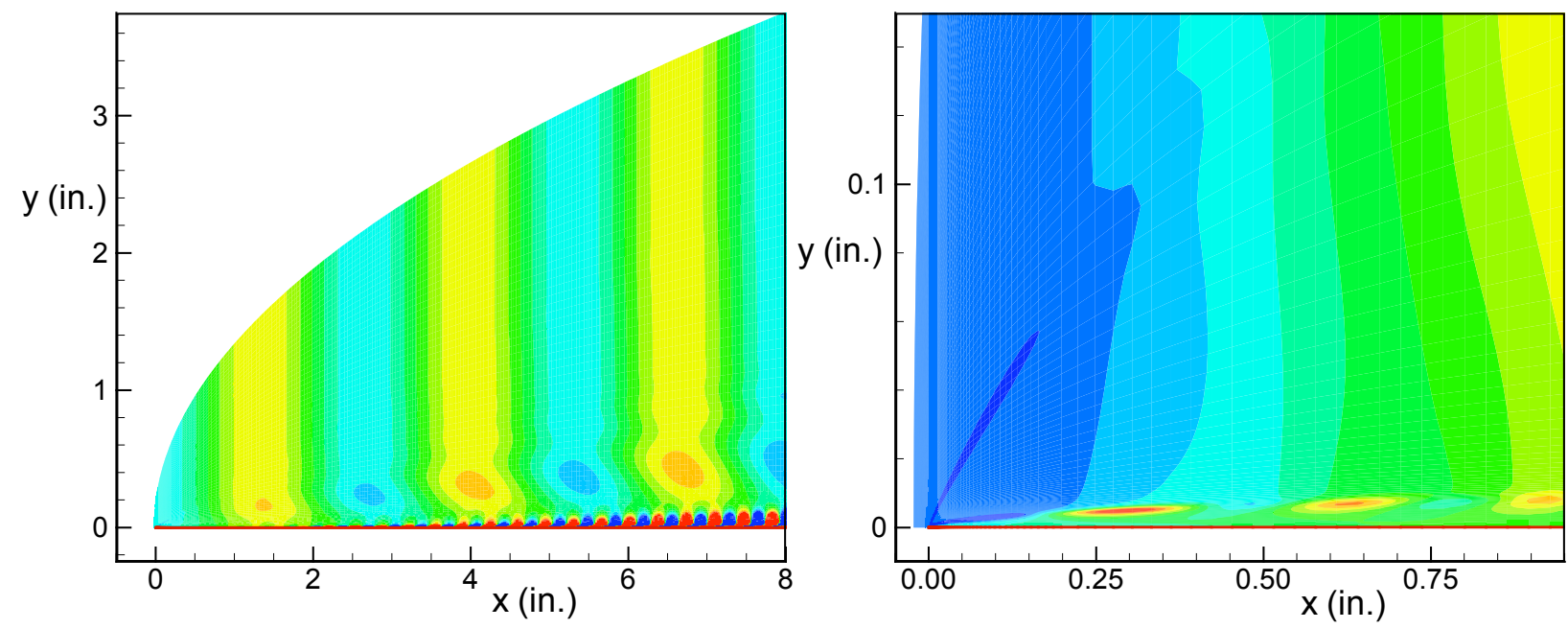

Figure 8. Contours of the unsteady density fluctuations due to the interaction of a 3D fast acoustic wave with a flat plate with a blunted leading edge. $F=1.25 * 10^{-4}, \beta=0.025$, incident angle 0.0 . 


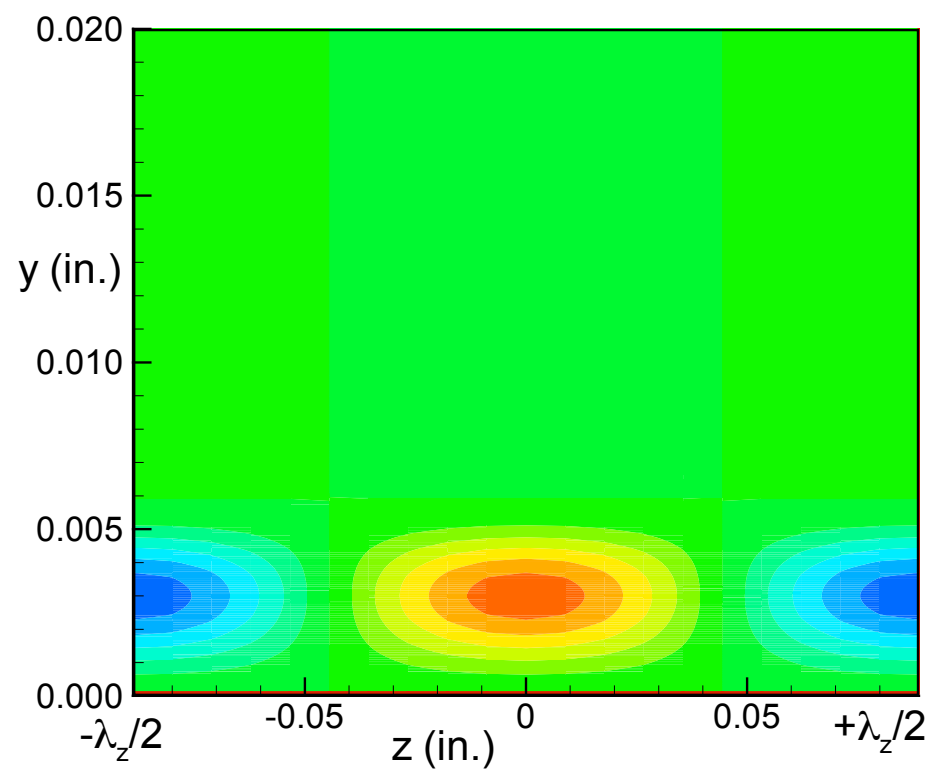

Figure 9. Contours of the $u$-velocity in the $y-z$ plane at $x=2.0$ inches.

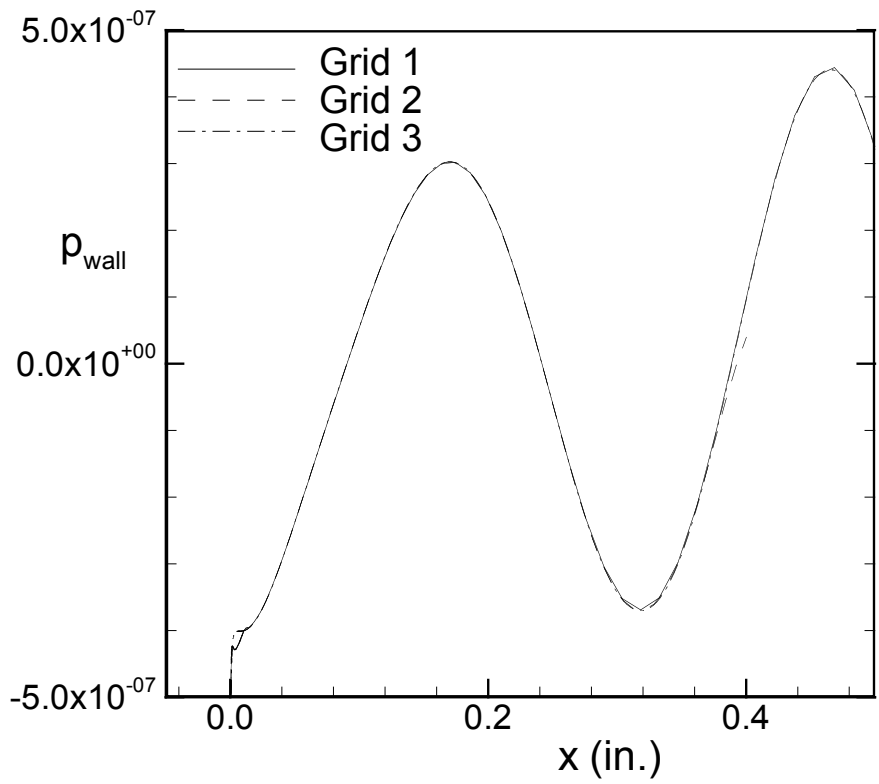

Figure 10. Pressure fluctuations along the wall near the nose region for three different grids. 

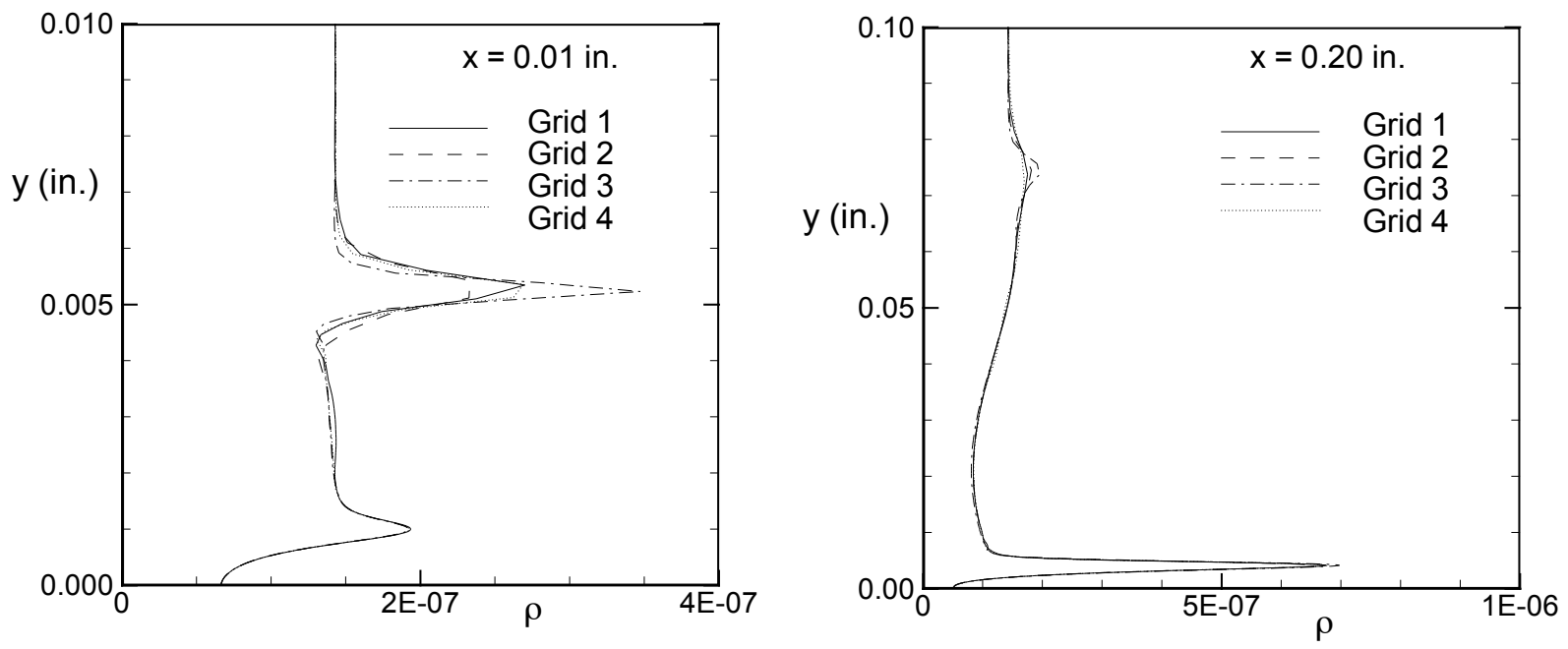

Figure 11. Eigenfunctions of the density fluctuations near the nose for three different grids.
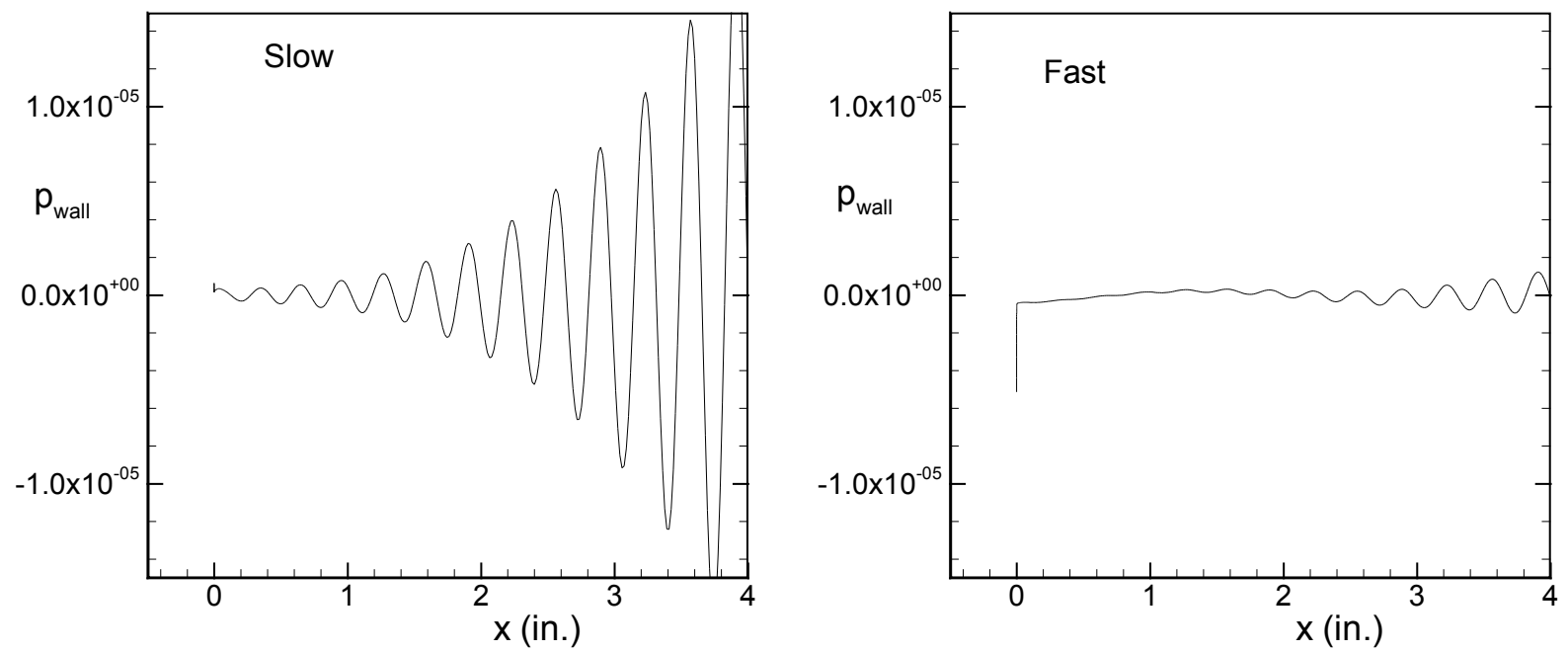

Figure 12. Pressure fluctuations along the wall for the slow and the fast waves. $\mathrm{F}=1.25^{*} 10^{-4}$, $\beta=\mathbf{0 . 0 2 5}$. 

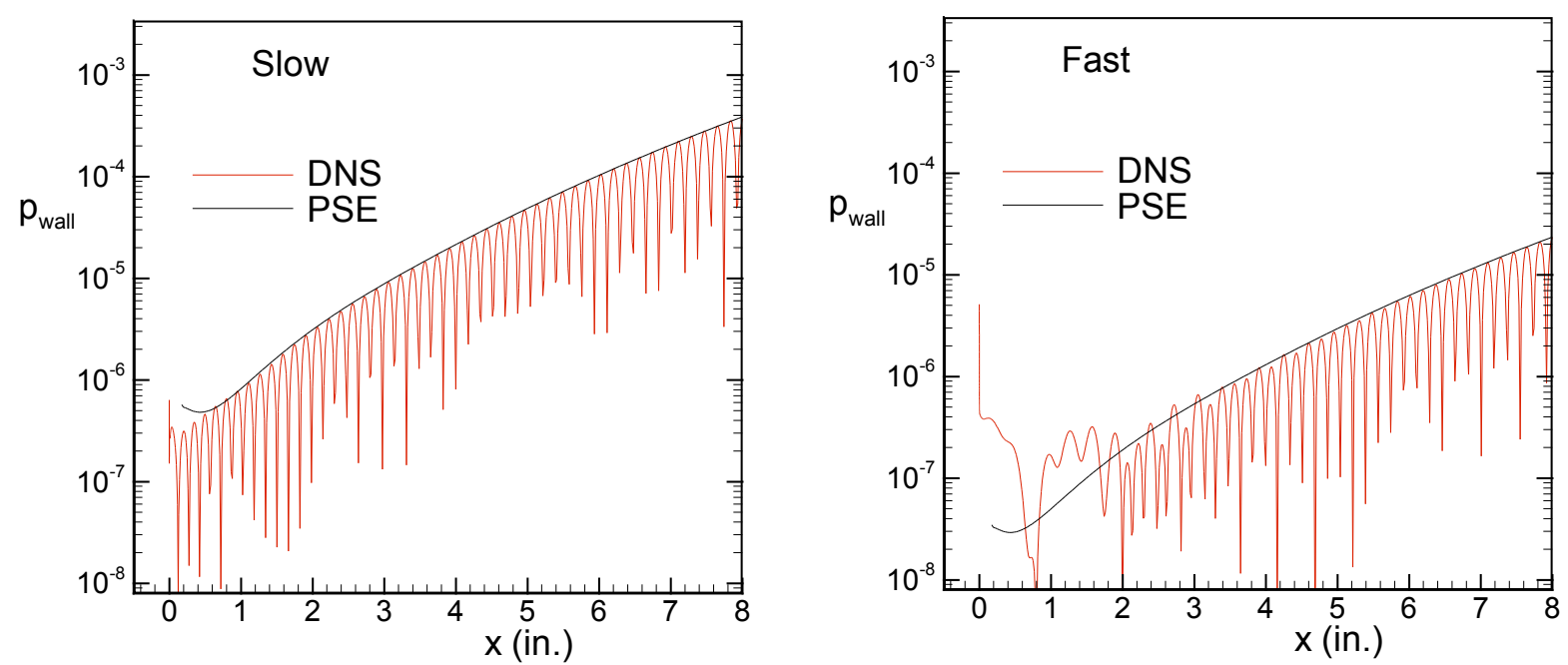

Figure 13. Amplitude of the pressure fluctuation at the wall and comparison with the PSE. $F=1.25 * 10^{-4}, \beta=0.025$.
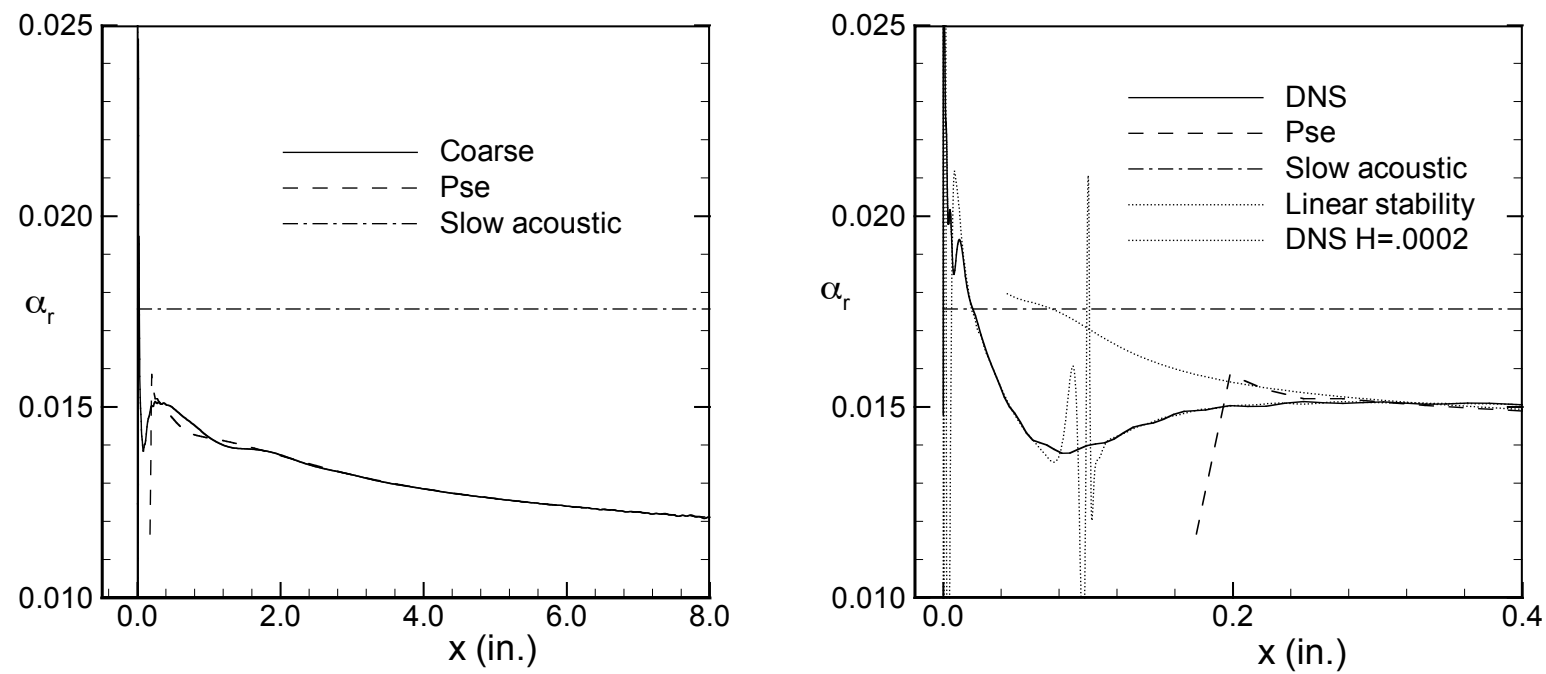

Figure 14. Wavenumber of the pressure fluctuation at the wall and comparison with the PSE $F=1.25 * 10^{-4}, \beta=0.025$. 

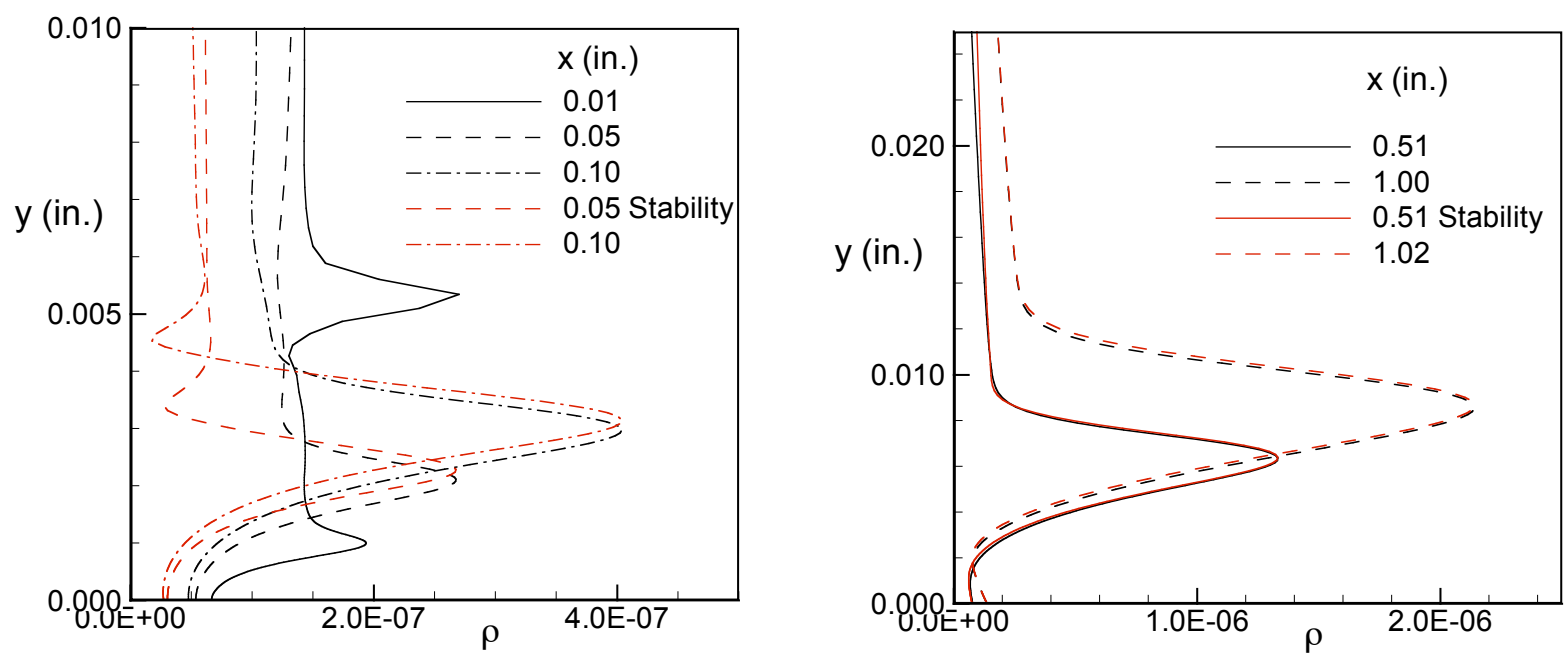

Figure 15. Eigenfunctions of the density fluctuations and comparison with the linear stability calculations. $F=1.25 * 10^{-4}, \beta=0.025$.

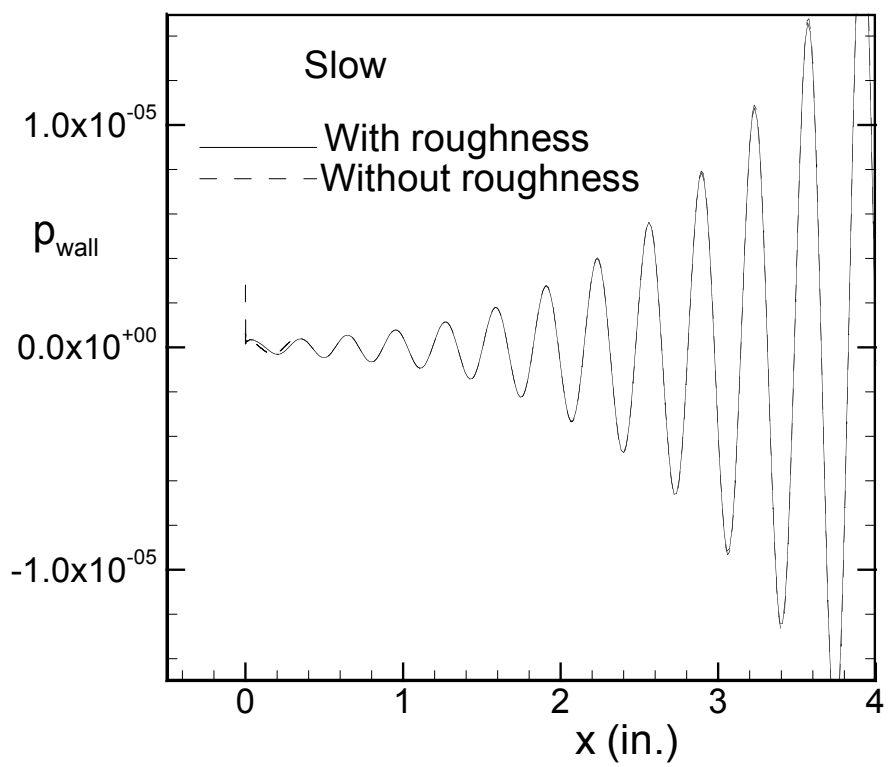

Figure 16. Pressure fluctuations along the wall for the slow wave with and without roughness. $F=1.25 * 10^{-4}, \beta=0.025$. 